\title{
The prevalence and associated factors of aspirin resistance among prophylactic aspirin users
}

\author{
Samaneh Ghorbani-Shirkouhi', Fatemeh Ashouri', Saeideh Aghayari Sheikh Neshin ${ }^{1}$, Alia Saberi², \\ Bahador Hasanzadeh ${ }^{1}$, Parisa Shahshahani ${ }^{3}$ \\ ${ }^{1}$ Neuroscience Research Center, School of Medicine, Guilan University of Medical Sciences, Rasht, Iran \\ ${ }^{2}$ Neuroscience Research Center, Department of Neurology, Poursina Hospital, School of Medicine, \\ Guilan University of Medical Sciences, Rasht, Iran \\ ${ }^{3}$ Department of Neurology, Poursina Hospital, School of Medicine, \\ Guilan University of Medical Sciences, Rasht, Iran
}

\begin{abstract}
Background. Aspirin is an antiplatelet used for the secondary prevention after vascular events. It is also suggested for the primary prevention of vascular events in high risk people, however, despite using standard prophylactic doses, aspirin resistance may result in therapeutic failure and arterial thrombosis. Since the prevalence of aspirin resistance and its associated factors were heterogeneous in different studies, this study was conducted to determine the prevalence and associated factors of aspirin resistance in an Iranian population under aspirin for primary prevention of vascular events.

Methods. 264 patients without documented vascular disease with $80 \mathrm{mg}$ daily aspirin consumption for at least one month enrolled in this cross-sectional study. Aspirin resistance was assessed by the measurement of thromboxane B2 in the urine samples using the enzyme-linked immunosorbent assay method. Aspirin resistance was defined as a urine level of thromboxane B2 $\geq 1700 \mathrm{ng} / \mathrm{dl}$.

Results. The prevalence of aspirin resistance in this study was $9.8 \%$. Age (OR $=0.935,95 \% \mathrm{Cl}: 0.880-0.993$, $\mathrm{P}=0.028)$ and geographical regions $(\mathrm{OR}=0.117,95 \% \mathrm{Cl}: 0.014-0.958, \mathrm{P}=0.045)$ showed independent correlation with aspirin resistance. No significant association observed between aspirin resistance and gender, diabetes mellitus, hypertension, hyperlipidemia, smoking, duration of aspirin use, body mass index, and drug history.

Conclusions. Despite the standard daily dose of aspirin for primary prevention of vascular events, some of our patients exhibited aspirin resistance that was directly related to a higher age and geographical region. More studies are required to clarify the beneficial role of aspirin resistance test before planning a preventive strategy in high risk individuals.
\end{abstract}

Keywords: aspirin, aspirin resistance, primary prevention, stroke, cardiovascular disease, urine thromboxane B2

\section{INTRODUCTION}

Cardiovascular and cerebrovascular disease are listed as the primary causes of death and disability globally (1). In addition to activation of coagulation system and thrombus formation, the inflammatory mechanisms have an essential role in the pathogenesis of acute arterial thrombosis and aggravation of tissue damage. Along with modification of conventional risk factors of vascular dis- ease (e.g. diabetes mellitus), targeting inflammation have been also proposed for the prevention of thrombotic vascular events (2). Platelets have been recognized as a major player of both coagulation and inflammation interconnecting the two systems in health and disease (3). This explains the role of daily low dose aspirin in the primary and secondary prevention of cardiovascular disease (4). 
Aspirin and non-steroidal anti-inflammatory drugs have been available for decades to relieve pain and inflammation (5). Aspirin irreversibly inhibits and modifies enzyme activity of cyclooxygenase (COX) (6). COX is an enzyme that converts arachidonic acid into prostaglandin $\mathrm{H} 2$, the major precursor of prostacyclin and thromboxane A2 (TXA2). Prostacyclin contributes to platelet disaggregation, anti-inflammation, adjustment of smooth muscle cell contraction and vasodilation. On the contrary, TXA2 that is produced largely by the platelets is a vasoconstrictor and facilitates platelet aggregation (7). The anti-thrombotic effect of aspirin is due to irreversible inhibition of TXA2 production for the entire half-life of platelets about 8 to 10 days (8). A low-dose aspirin (50 and 100 $\mathrm{mg}$ /day) almost completely inhibits production of TXA2 by COX-1 (9) and in high risk patient for vascular events aspirin shown to reduce risk of serious vascular events up to $25 \%$ (10). However, not all individuals are completely responsive to therapy and develop vascular events while consuming standard daily dose of aspirin. This treatment failure is the clinical definition of aspirin resistance but there are also laboratory definitions described as inability of aspirin to cause platelet dysfunction or inability to inhibit TXA2 production by COX-1 (11). Prevalence of aspirin resistance in various populations using different laboratory methods was heterogeneous and reported up to $70 \%$ (12). Associated factors with aspirin resistance are also varied in different reports and the most prevalent reported factors are noncompliance, patients' clinical conditions, drug interactions, genetic polymorphism in contributing genes such as COX-1 and COX-2 (13-15).

Different methods have been introduced to diagnose aspirin resistance including light transmission aggregometry with arachidonic acid or adenosine diphosphate, platelet function analyzer test, VerifyNow Aspirin test, thromboelastography and measurements of serum and urine TXB2 metabolites $(16,17)$. Serum TXA2 is mainly produced by platelets and rapidly hydrolyzes to its inactive metabolite TXB2 with a long half-life and is easily excreted into the urine. Since the kidney does not produce TXB2 therefore it is a reliable indicator of TXA2 production $(18,19)$. Measurement of urinary TXB2 metabolite, 11-dehydrothromboxane B2 (11dhTxB2) levels with $62.2 \%$ sensitivity and
$82.2 \%$ specificity is a standard method to evaluate the aspirin resistance (16).

Because of the importance of aspirin resistance and the variation of its prevalence and associated factors in different studies, the purpose of this study is to assess the prevalence of aspirin resistance among subjects without a history of vascular events under aspirin for primary prevention. We also aimed to evaluate the associated factors including the demographic variables, comorbidities, concomitant drugs in the population from the Guilan Province in the north of Iran.

\section{MATERIAL AND METHODS}

\section{Participants and study design}

This cross-sectional study was approved by the Ethics Committee of the Guilan University of Medical Sciences (Ethics Code: IR.GUMS. REC.1396.97). 264 patients who were under 80 $\mathrm{mg}$ daily aspirin for at least one month consecutively recruited to the study from neurology clinics affiliated with the Guilan University of Medical Sciences. All the subjects were given written informed consent before participating in the study. None of the subjects had a history of taking other anti-platelets, anticoagulants or non-steroidal anti-inflammatory drugs (NSAIDs) in the recent 10 days. Included patients were using aspirin for primary prevention without history of stroke, myocardial infarction or any vascular thrombotic event. Patient with liver and renal failure were excluded from the study. History of hypertension, diabetes mellitus, hyperlipidemia, and smoking were documented for each subject. Concomitant drug history was taken from all patients and drugs that were used regularly documented for each patient. Other documented variables were age, sex, body mass index (BMI), duration of aspirin intake (year). To decrease the impact of genetic polymorphism in the platelet glycoproteins and COX genes that have been associated with aspirin resistance $(20,21)$ geographical regions of Guilan province were categorized into center, east, west, south and north and the subjects distributed in each group if both parents were belonged to the same region.

\section{Sample size}

Akay OM et al. (22) estimated 27.5\% prevalence for aspirin resistance in healthy subjects 
(22). With the accuracy of 0.06 and the type 1 error 0.05 , the sample size was calculated to be 213 . Considering five persons for each variable, the number of 263 was determined.

$$
\begin{aligned}
& \mathrm{N}=\left(\mathrm{Z}_{1-\alpha / 2}\right)^{2}[\mathrm{p}(1-\mathrm{p})] / \mathrm{d}^{2} \\
& \mathrm{~N}=\frac{1.96^{2}[0.275(1-0.275)]}{(0.06)^{2}}=212.75=213
\end{aligned}
$$

\section{Measurement of urinary $11 \mathrm{dhTXB2}$}

First morning urine samples were stored in $-80^{\circ} \mathrm{C}$ until analysis, and urine level of $11 \mathrm{dhTXB} 2$ was determined using commercially available ELISA kits (EASTBIOPHARM, USA), and the $11 \mathrm{dhTXB} 2$ levels were normalized to urinary creatinine level. Aspirin resistance was defined urine level of $11 \mathrm{dhTXB} 2 \geq 1700 \mathrm{ng} / \mathrm{dl}$ based on the definition in the laboratory kit.

\section{Data analysis}

Mean \pm standard deviation of continuous variables was calculated and Student's t tests were used for analysis of normal variables. Non-normal variables were analyzed using Mann-Whitney U test.
The chi-squared test and Fisher's exact tests applied for the analysis of proportions of categorical variables. Logistic regression model was used to examine prediction of aspirin resistance by age, BMI, and region. Statistical analysis was performed using the SPSS statistical analysis software v.16.0 (SPSS, Chicago, Illinois, USA) and statistical significance of $\mathrm{P} \leq 0.05$ was applied to all tests.

\section{RESULTS}

Total 264 subjects with at least one month of 80 $\mathrm{mg}$ aspirin usage were included in the study. The average age of subjects was $62 \pm 11.75$ years (39-90 years), including 109 men (41\%) and 155 women (51\%). The frequency of aspirin resistance in study population was $9.8 \%$ (26 subjects). Table 1 shows the characteristics of the subjects and the frequency of aspirin resistance for each group.

The average age of patients with aspirin resistant was $69.8 \pm 9.8$ years and they were significantly older than non-resistant patients with average age of $61.7 \pm 11.7(p=0.024)$. The average of BMI was $24.9 \pm 3.7$ in aspirin resistant group and $26.5 \pm 5$ in

\begin{tabular}{|c|c|c|c|c|}
\hline & & $\begin{array}{c}\text { Total number } \\
\mathrm{N}(\%)\end{array}$ & $\begin{array}{c}\text { Resistant to aspirin } \\
\mathrm{N}(\%)\end{array}$ & $p$-value \\
\hline \multirow[t]{2}{*}{ Gender } & male & $109(41 \%)$ & $8(7.3 \%)$ & \multirow[t]{2}{*}{0.43} \\
\hline & female & $155(59 \%)$ & $18(11.6 \%)$ & \\
\hline \multirow{3}{*}{ Age } & $<55$ years & $70(26 \%)$ & $2(2.9 \%)$ & \multirow[t]{3}{*}{0.231} \\
\hline & $55-65$ years & $94(36 \%)$ & $10(10.6 \%)$ & \\
\hline & $\geq 65$ years & $100(38 \%)$ & $14(14 \%)$ & \\
\hline \multirow{3}{*}{ Body mass index (BMI) } & $\mathrm{BMI}<25$ & $119(45 \%)$ & $16(13.4 \%)$ & \multirow[t]{3}{*}{0.161} \\
\hline & $25 \leq \mathrm{BMI}<30$ & $96(36 \%)$ & $10(10.4 \%)$ & \\
\hline & $\mathrm{BMI} \geq 30$ & $49(19 \%)$ & $0(0 \%)$ & \\
\hline \multirow[t]{2}{*}{ Diabetes mellitus } & yes & $126(48 \%)$ & $12(9.5 \%)$ & \multirow{2}{*}{$\begin{array}{c}1.000 \\
\text { (Fisher's exact test) }\end{array}$} \\
\hline & no & $138(52 \%)$ & $14(10.1 \%)$ & \\
\hline \multirow[t]{2}{*}{ Hyperlipidemia } & yes & $118(45 \%)$ & $10(8.5 \%)$ & \multirow{2}{*}{$\begin{array}{c}0.431 \\
\text { (Fisher's exact test) }\end{array}$} \\
\hline & no & $146(55 \%)$ & $16(11.0 \%)$ & \\
\hline \multirow[t]{2}{*}{ Hypertension } & yes & $189(71 \%)$ & $18(9.5 \%)$ & \multirow[t]{2}{*}{0.175} \\
\hline & no & $75(29 \%)$ & $7(9.4 \%)$ & \\
\hline \multirow[t]{2}{*}{ Smoking } & yes & $31(12 \%)$ & $2(6.4 \%)$ & \multirow{2}{*}{$\begin{array}{c}1.000 \\
\text { (Fisher's exact test) }\end{array}$} \\
\hline & no & & $24(10.3 \%)$ & \\
\hline \multirow{5}{*}{$\begin{array}{l}\text { Geographical Distribution in } \\
\text { Guilan Province (Region) }\end{array}$} & Center & $91(34 \%)$ & $2(2.2 \%)$ & \multirow[t]{5}{*}{0.026} \\
\hline & East & $42(16 \%)$ & $4(9.5 \%)$ & \\
\hline & West & $46(17 \%)$ & $10(21.7 \%)$ & \\
\hline & South & $33(13 \%)$ & $0(0 \%)$ & \\
\hline & North & $52(20 \%)$ & $10(19.2 \%)$ & \\
\hline \multirow{4}{*}{ Duration of taking aspirin (year) } & Less than 1 year & $47(18 \%)$ & $47(100 \%)$ & \multirow[t]{4}{*}{0.289} \\
\hline & $1-5$ years & $138(52 \%)$ & $120(87 \%)$ & \\
\hline & $5-10$ years & $51(19 \%)$ & $47(92.2 \%)$ & \\
\hline & Over 10 years & $28(11 \%)$ & $24(85.7 \%)$ & \\
\hline
\end{tabular}

TABLE 1. Baseline characteristics of study participants 
non-resistant without significant difference between groups $(p=0.286)$. Age and BMI of subjects were subcategorized that showed in table 1 . No significant difference existed between frequency of aspirin resistance and diabetes mellitus, hyperlipidemia, hypertension, smoking, and duration of aspirin consumption.

Table 1 shows the distribution of the individuals based on their geographical regions and the frequency of aspirin resistance in each group and the results showed a significant difference between the groups $(\mathrm{p}=0.026)$. Most of the patients were from center (34\% of total number) but they showed $2.2 \%$ aspirin resistance however, patients from west had lower frequency (17\% from total patients) they showed $21.7 \%$ aspirin resistance which was significantly higher than other groups.

Table 2 shows the resistance to aspirin based on concomitant drug history while none of the drugs was significantly related to aspirin resistance.

TABLE 2. Frequency of aspirin resistance according to drug usage

\begin{tabular}{|l|c|c|c|c|}
\hline \multicolumn{1}{|c|}{ Drug groups } & \multicolumn{2}{|c|}{$\begin{array}{c}\text { Frequency of aspirin } \\
\text { resistance }\end{array}$} & $\begin{array}{c}\text { Total drug } \\
\text { users } \\
\text { (N) }\end{array}$ & P value \\
\cline { 2 - 3 } & $\begin{array}{c}\text { Drug } \\
\text { users } \\
\%\end{array}$ & $\begin{array}{c}\text { Non-drug } \\
\text { users } \\
\%\end{array}$ & & \\
\hline BB & $9.3 \%$ & $10.1 \%$ & 86 & 1.000 \\
\hline CCB & $12.5 \%$ & $9.5 \%$ & 32 & 0.658 \\
\hline ARB & $10.4 \%$ & $9.5 \%$ & 96 & 1.000 \\
\hline ACEi & $9.5 \%$ & $9.9 \%$ & 21 & 1.000 \\
\hline $\begin{array}{l}\text { Cholesterol } \\
\text { lowering drug }\end{array}$ & $11.7 \%$ & $7.9 \%$ & 137 & 0.563 \\
\hline $\begin{array}{l}\text { Triglyceride } \\
\text { lowering drug }\end{array}$ & $11.8 \%$ & $9.7 \%$ & 17 & 0.574 \\
\hline $\begin{array}{l}\text { Liver enzyme } \\
\text { inhibitor } \\
\text { anticonvulsants }\end{array}$ & 0 & $10.2 \%$ & 10 & 1.000 \\
\hline $\begin{array}{l}\text { Liver enzyme } \\
\text { inducing } \\
\text { anticonvulsants }\end{array}$ & $14.3 \%$ & $9.6 \%$ & 14 & 0.525 \\
\hline $\begin{array}{l}\text { Glucose lowering } \\
\text { drug }\end{array}$ & $10.1 \%$ & $9.7 \%$ & 99 & 1.000 \\
\hline Nitroglycerin & $14.3 \%$ & $9.3 \%$ & 28 & 0.629 \\
\hline
\end{tabular}

$N$ - number; $B B$ - beta blocker; $C C B$ - calcium channel blocker; $A R B$ - angiotensin receptor blocker; $A C E i$ - angiotensin converting enzyme inhibitor

In multivariate regression analysis, variables with p-value less than 0.25 (region, age and BMI) entered the regression model. Finally, region ( $\mathrm{p}=$ $0.049)$ and age $(p=0.028)$ were independently correlated with aspirin resistance. The aspirin resistance in people from out of the center was 8.3 times higher than those from center $(\mathrm{OR}=8.267$,
95\% CI: 1.01-67.58). Moreover, with increasing age, aspirin resistance significantly increased about 1.070 folds each year $(\mathrm{p}=0.028$, OR: 1.070, 95\% CI: 1.007-1.137).

TABLE 3. Multivariate regression analysis and estimated odds ratio for variables affecting aspirin resistance

\begin{tabular}{|c|c|c|c|c|c|c|}
\hline & B & SE & Sig. & $\operatorname{Exp}(\mathrm{B})$ & \multicolumn{2}{|c|}{$95 \% \mathrm{Cl}$ for $\operatorname{Exp}(\mathrm{B})$} \\
\cline { 5 - 7 } & & & & & Lower & Upper \\
\hline age & 0.068 & 0.031 & 0.028 & 1.070 & 1.007 & 1.137 \\
\hline BMI & -0.073 & 0.072 & 0.311 & 0.929 & 0.807 & 1.071 \\
\hline region & 2.112 & 1.072 & 0.049 & 8.267 & 1.011 & 67.576 \\
\hline Constant & -8.555 & 3.475 & 0.014 & 0.000 & & \\
\hline
\end{tabular}

$B M I$ - body mass index; SE - standard error; $\mathrm{Cl}$ - confidence interval

\section{DISCUSSION}

The frequency of aspirin resistance among prophylactic aspirin users was $9.8 \%$ in this study. Gender, diabetes mellitus, hyperlipidemia, hypertension, smoking, duration of aspirin consumption and BMI showed no association with aspirin resistance. Age and geographical region were independently associated with aspirin resistance.

Most studies used inconsistent laboratory methods and populations with our study making it difficult to compare the results. However, we briefly review their results to show the prevalence of aspirin resistance in different populations with standard but different doses of aspirin.

Salama et al. assessed aspirin resistance by optical platelet aggregometry and serum TXB2 level in patients with coronary artery disease. Patients were under $150 \mathrm{mg}$ daily aspirin and $22 \%$ of them were aspirin resistant higher than the prevalence observed in our subjects. With doubling the dose of aspirin in resistant patients to $300 \mathrm{mg}$ daily enhanced therapeutic response was detected. Furthermore, they reported a significant correlation between aspirin resistance and diabetes mellitus, obesity, and dyslipidemia that were inconsistent with our findings (17). Sadeghi et al. studied 170 patients with coronary artery stenosis taking daily aspirin and using TXB2 level in urine and reported $75.3 \%$ aspirin resistance in an Iranian population in a different region from the present study (14). The significant difference between aspirin resistance in Sadeghi study and our subjects may be highly related to the presence of coronary artery disease in their participants. According to previous studies prevalence of aspirin resistance was signif- 
icant among patients with stable coronary heart disease (up to $70 \%$ ) and acute myocardial infarction (up to $80 \%$ ) (23).

Liu et al. studied the prevalence of aspirin resistance in 469 elderly patients with cardiovascular disease and metabolic syndrome receiving 75$100 \mathrm{mg} / \mathrm{d}$ near to the dose of aspirin in our subjects, with the Light Transmission Aggregometry test. They reported that $8.1 \%$ of patients were aspirin resistant and metabolic syndrome was a significant risk factor (24). Another study by Akay et al. measured aspirin resistance by platelet aggregometry in 280 healthy volunteers receiving $100 \mathrm{mg} / \mathrm{d}$ aspirin and $27.5 \%$ of patients were aspirin-resistant. Age, $\mathrm{ESR}$, and $\mathrm{HbA} 1 \mathrm{c}$ levels were higher in aspirin-resistant individuals. In this study which measured aspirin resistance in a primary prevention population the same as our study, resistance was higher than our study that could be mainly due to the different diagnostic test (22). One study by Azmin et al. on 50 patients with acute stroke who took 50 $\mathrm{mg}$ of aspirin per day for 5 days before the test, showed a $14 \%$ resistance to aspirin. They also reported that low HDL levels were associated with resistance to aspirin but no correlation existed between aspirin resistance and total cholesterol, triglyceride, LDL, HbA1c and AST, ALT, ALP, urea and creatinine levels in serum, and smoking (25). One study by Üsküdar Cansu et al. evaluated the aspirin resistance among 175 healthy men who received at least 7 days of $100 \mathrm{mg}$ aspirin. A percentage of $28 \%$ of participants were resistant, $62 \%$ were semi-resistant, and $10 \%$ were susceptible to aspirin. Similar to our study they observed a significant correlation with increasing age (26). Habizal et al. studied 69 diabetic patients receiving daily aspirin and presented $17.4 \%$ for the prevalence of aspirin resistance, but the levels of $\mathrm{HbAlc}$ and inflammatory markers were not correlated with aspirin resistance (27). Another study of 130 non-diabetic and diabetic subjects conducted by Toptas et al. showed resistance to aspirin in $41.9 \%$ of the diabetic patients and high blood cholesterol level was the only predictor of aspirin resistance (28). It seems that aspirin resistance in our population was lower than other studies and resistance to aspirin in most studies were between $10 \%$ and $25 \%$.

In the present study, the role of diabetes mellitus, hypertension, hyperlipidemia, age, sex, and duration of aspirin consumption were assessed.
However, none of these factors affected aspirin resistance. In the logistic regression analysis, the effect of three variables of age, BMI and region was studied, and finally, age and region remained as correlated factors. BMI was not correlated with aspirin resistance in our study whereas obesity (17) and metabolic syndrome (24) were associated with aspirin resistance in other studies.

As mentioned earlier, another factor examined in this study was the geographical region of individuals. It seems that people from the center of the Guilan province were less resistant to aspirin than those from the other regions and people from the west showed higher resistance. However, the study was conducted in a small geographic area with genetic similarities but the results have raised questions for further research on the genetic basis or other related factors to the observed difference. Considering the significantly different prevalence in another study from Iranian population (14), more studies with consistent laboratory methods are needed to clear the contributing factors.

We have also investigated the relationship between concurrent drug consumption with aspirin that did not show any significant relationship with aspirin resistance. In agreement with our results, in a study on patients with recurrent ischemic stroke Pobas et. al. reported that neither hypertension nor antihypertensive drugs were associated with aspirin resistance and they observed a $20.5 \%$ of aspirin resistance among the patients (29). In this study lipid and triglyceride lowering agents did not show relationship with aspirin resistance however in a study on 178 patients with stable coronary artery disease that $11.2 \%$ of them were resistant to aspirin, 3 months of statin therapy significantly reduced aspirin resistance (30). The association of statins and lower prevalence of aspirin resistance was also observed by Al-Azzam and colleagues in a group of 418 patients with $18.7 \%$ aspirin resistance (31). Feher et. al. also observed a significant role for concomitant drug use. They reported that users of beta-blockers and angiotensin converting enzyme inhibitors had a lower frequency of aspirin resistance while statin was more frequently used by patients with aspirin resistance. The logistic regression model in their study showed that statin was the only drug predicting aspirin resistance (32). In conclusion, it seems that the inconsistency in the contributing factors with aspirin resistance also exists for drug history. 
Our study has some limitations. We have assessed aspirin resistance using urine TXB2 level with $62.2 \%$ sensitivity therefore the prevalence of aspirin resistance may have been underestimated. We performed the study with only one test and did not examine the results with another method while inconsistency between different methods has been detected (33). Moreover, we preformed the study once while evidence showed the unpredictability of aspirin resistance over time (33).

\section{CONCLUSIONS}

The prevalence of aspirin resistance in our study although seems low yet is significant enough to pay attention. Elevated age was related to higher aspirin resistance in our patients therefore older patients that are already at higher risk for arterial

\section{REFERENCES}

1. Benjamin EJ, Muntner $P$, Alonso A et al. Heart disease and stroke Statistics-2019 update a report from the American Heart Association. Circulation. 2019 Mar 5;139(10):e56-28.

2. Kelly PJ, Murphy S, Coveney $S$ et al. Anti-inflammatory approaches to ischaemic stroke prevention. J Neurol Neurosurg Psychiatry. 2018;89(2):211-8.

3. Hottz ED, Monteiro AP, Bozza FA, Bozza PT. Inflammasome in platelets: allying coagulation and inflammation in infectious and sterile diseases? Mediators Inflamm. 2015 Feb 26;2015.

4. Ittaman SV, VanWormer JJ, Rezkalla SH. The role of aspirin in the prevention of cardiovascular disease. Clin Med Res. 2014 Dec 1;12(3-4):147-54.

5. Moyad MA. An introduction to aspirin, NSAIDs, and COX-2 inhibitors for the primary prevention of cardiovascular events and cancer and their potential preventive role in bladder carcinogenesis: part I. Semin Urol Oncol. 2001 Nov 1;19(4):294-305.

6. Goel A, Aggarwal S, Partap S et al. Pharmacokinetic solubility and dissolution profile of antiarrythmic drugs. Int J Pharma Prof Res. 2012;3(1):592-601.

7. Belton O, Byrne D, Kearney D et al. Cyclooxygenase-1 and-2dependent prostacyclin formation in patients with atherosclerosis. Circulation. 2000 Aug 22;102(8):840-5.

8. Mainous III AG, Tanner RJ, Shorr RI, Limacher MC. Use of aspirin for primary and secondary cardiovascular disease prevention in the United States, 2011-2012. J Am Heart Assoc. 2014 Jul 14;3(4):e000989.

9. Warner TD, Nylander S, Whatling C. Anti-platelet therapy: cyclo-oxygenase inhibition and the use of aspirin with particular regard to dual anti-platelet therapy. Br J Clin Pharmacol. 2011 Oct;72(4):619-33.

10. Antithrombotic Trialists' Collaboration. Collaborative meta-analysis of randomised trials of antiplatelet therapy for prevention of death, myocardial infarction, and stroke in high risk patients. BMJ. 2002 Jan 12;324(7329):71-86.

11. Patrono C. Aspirin resistance: definition, mechanisms and clinical read-outs. J Thromb Haemost. 2003 Aug;1(8):1710-3.

12. Kasotakis G, Pipinos II, Lynch TG. Current evidence and clinical implications of aspirin resistance. J Vasc Surg. 2009 Dec 1;50(6):1500-10.

13. Mehta JL, Mohandas B. Aspirin resistance: Fact or fiction? A point of view. World J Cardiol. 2010 Sep 26;2(9):280. events might be good candidates to go under aspirin resistance assessment in clinical practice. Therapeutic failure of antiplatelet agents is a complication that may result in serious vascular events and standard guidelines are required based on data from various populations. We believe that the results of this study have increased current data regarding the aspirin resistance and its associated factors.

\section{Acknowledgement}

We would like to thank all the participants, and all the staffs of the neurology clinic of Poursina hospital.

\section{Authors' contributions}

All authors participated in study design, data collection, analysis and drafting the manuscript.

14. Sadeghi M, Emami A, Ziyaei $\mathrm{N}$ et al. Aspirin resistance and ischemic heart disease on Iranian experience. Adv Biomed Res. 2012;1:33.

15. Schwartz KA. Aspirin resistance: a clinical review focused on the most common cause, noncompliance. Neurohospitalist. 2011 Apr;1(2):94-103.

16. Blais N, Lordkipanidze M, Pharand C et al. Normal Response to Aspirin in Healthy Individuals: Cross-Comparison of Light Transmission Aggregometry, VerifyNow ${ }^{\mathrm{TM}}$ System, Platelet Count Drop, Thrombelastography (TEG®) and Urinary 11-dehydrothromboxane B2. Blood. 2007; 110 (11): 934.

17. Salama M, Mohamed Morad AR, Saleh M et al. Resistance to low-dose aspirin therapy among patients with acute coronary syndrome in relation to associated risk factors. J Clin Pharm. 2012 Dec;37(6):630-6.

18. Cattaneo M. Resistance to antiplatelet drugs: molecular mechanisms and laboratory detection. J Thromb Haemost. 2007 Jul:5:230-7.

19. Lordkipanidzé M, Pharand C, Schampaert E et al. A comparison of six major platelet function tests to determine the prevalence of aspirin resistance in patients with stable coronary artery disease. Eur Heart J. 2007 Jul 1;28(14):1702-8.

20. Sharma V, Kaul S, Al-Hazzani A et al. Association of COX-2 rs20417 with aspirin resistance. J Thromb Thrombolysis. 2013 Jan; 35(1):95-9.

21. Wang $H$, Sun $X$, Dong $W$ et al. Association of GPI a and COX-2 gene polymorphism with aspirin resistance. J Clin Lab Anal. 2018 May;32(4):e22331.

22. Akay OM, Canturk $Z$, Akin $E$ et al. Aspirin-resistance frequency: a prospective study in 280 healthy Turkish volunteers. Clin Appl Thromb Hemost. 2009 Feb;15(1):98-102.

23. Zimmermann $\mathrm{N}$, Hohlfeld T. Clinical implications of aspirin resistance. Thromb Haemost. 2008;100(09):379-90.

24. Liu L, Gao Y-H, Cao J et al. High prevalence of aspirin resistance in elderly patients with cardiovascular disease and metabolic syndrome. J Geriatr Cardiol. 2016 Sep;13(6):531.

25. Azmin S SR, Rabani R, Nafisah WY et al. Biochemical aspirin resistance in stroke patients-a cross-sectional single centre study. EXCLI journal. 2013;12:907-15.

26. Cansu DÜ, Akay OM, Cantürk Z, Gülbafl Z. Aspirin resistance frequency in healthy males. Turk J Hematol. 2008 Jun 5;25:83-6. 
27. Habizal NH, Halim SA, Bhaskar S et al. Prevalence of aspirin resistance in diabetic patients and its associated factors. Malays $\mathrm{J}$ Med Sci. 2015 Jan;22(1):50

28. Toptas T, Tasdemir E, Demir $C$ et al. Author's reply: Predictors of aspirin resistance. Ups J Med Sci. 2014 Aug 1;119(3):294-

29. Pobas FM, Pramantara IDP, Pinzon RT, Endro A. The Relations of Aspirin Resistance and Hypertension in Patients with Recurrent Ischemic Stroke. Int J Pharm Clin. 2015;7(6):402-5.

30. Tirnaksiz E, Pamukcu B, Oflaz H, Nisanci Y. Effect of high dose statin therapy on platelet function; statins reduce aspirin resistant platelet aggregation in patients with coronary heart disease. $J$ Thromb Thrombolysis. 2009 Jan;27(1):24-8

31. Al-Azzam SI, Alzoubi KH, Khabour O et al. The prevalence and factors associated with aspirin resistance in patients premedicated with aspirin. Acta Cardiol. 2012 Aug 1;67(4):445-8.

32. Feher G, Koltai K, Papp E et al. Aspirin resistance. Drugs Aging. $2006 \mathrm{Jul} ; 23(7): 559-67$.

33. Muir AR, McMullin MF, Patterson C, McKeown PP. Assessment of aspirin resistance varies on a temporal basis in patients with ischaemic heart disease. Heart. 2009 Aug 1;95(15):1225-9

\section{Conflict of interest: none declared}

Financial support: none declared 